\title{
Dilepton production at SPS and RHIC energies
}

\section{O. Linnyk*}

Institut für Theoretische Physik, Universität Gießen, 35392 Gießen, Germany

E-mail: Linnykefias.uni-frankfurt.de

\section{E. L. Bratkovskaya}

Institut für Theoretische Physik, Universität Frankfurt, 60438 Frankfurt am Main, Germany

\section{W. Cassing}

Institut für Theoretische Physik, Universität Gießen, 35392 Gießen, Germany

\begin{abstract}
The microscopic parton-hadron-string dynamics (PHSD) transport approach describes the full evolution of relativistic heavy-ion collisions: from the initial hard scatterings to the partonic phase in the early hot and dense reaction region (when the local energy density is above $\approx 1 \mathrm{GeV} / \mathrm{fm}^{3}$ ) followed by hadronization and off-shell hadron propagation and interactions. The description of quarks and gluons in PHSD is based on the dynamical quasiparticle model (DQPM) matched to reproduce lattice QCD results in thermodynamic equilibrium. The DQPM describes QCD properties in terms of single-particle Green's functions and leads to the notion of effective quasiparticles which are massive and have broad spectral functions (due to large interaction rates). Dressing the quark and gluon lines with effective propagators provided by the DQPM, we obtain cross sections of dilepton production in the reactions $q+\bar{q}->l^{+} l^{-}$(Born mechanism), $q+\bar{q}->l^{+} l^{-}+g$ (quark annihilation with the gluon Bremsstrahlung in the final state), and $q / \bar{q}+g->l^{+} l^{-}+q / \bar{q}$ (gluon Compton scattering) by off-shell quarks and gluons in the strongly interacting partonic phase. After implementing the off-shell cross sections for the partonic processes into the PHSD transport approach, we address the dilepton production from the parton interactions in the early stage of relativistic heavy-ion collisions at SPS and RHIC energies. Dilepton production in In+In collisions at $158 \mathrm{AGeV}$ and in $A u+A u$ at $\sqrt{s}=200 \mathrm{GeV}$ is calculated. By comparing to the data of the NA60 and PHENIX Collaborations, we study the relative importance of different dilepton production mechanisms and point out the regions in phase space where partonic channels are dominant.
\end{abstract}

XLIX International Winter Meeting on Nuclear Physics, BORMIO2011

January 24-28, 2011

Bormio, Italy

\footnotetext{
*Speaker.
} 


\section{The PHSD approach to dilepton radiation}

Early on, dileptons have been suggested as probes of the quark-gluon plasma (QGP) that is expected to be produced during the early stage of heavy-ion collisions at Super-Proton-Synchrotron

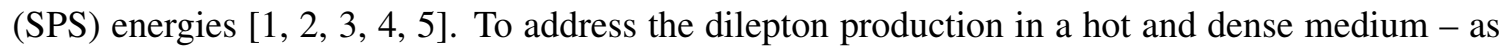
created in heavy-ion collisions - we employ an up-to-date relativistic transport model, i.e. the Parton Hadron String Dynamics [Q] (PHSD) that incorporates the explicit partonic phase in the early reaction region. Within PHSD, one solves generalized transport equations on the basis of the off-shell Kadanoff-Baym equations for Greens functions in phase-space representation (in the first order gradient expansion, beyond the quasiparticle approximation). The approach consistently describes the full evolution of a relativistic heavy-ion collision from the initial hard scatterings and string formation through the dynamical deconfinement phase transition to the quark-gluon plasma (QGP) as well as hadronization and to the subsequent interactions in the hadronic phase.

In the hadronic sector PHSD is equivalent to the Hadron-String-Dynamics (HSD) transport approach [ए], [1, प2] that has been used for the description of $p A$ and $A A$ collisions from SIS to RHIC energies and has lead to a fair reproduction of hadron abundances, rapidity distributions and transverse momentum spectra. In particular, HSD incorporates off-shell dynamics for vector mesons - according to Refs. [ए3] - and a set of vector-meson spectral functions [प14] that covers possible scenarios for their in-medium modification. Various models predict that hadrons change in the (hot and dense) nuclear medium; in particular, a broadening of the spectral function or a mass shift of the vector mesons has been expected. Furthermore, QCD sum rules indicated that a mass shift may lead to a broadening and vice versa [ㄷ]]; therefore both modifications should be studied simultaneously. In the off-shell transport, hadron spectral functions change dynamically during the propagation through the medium and evolve towards the on-shell spectral function in the vacuum.

The description of quarks and gluons in PHSD is based on a dynamical quasiparticle model for partons matched to reproduce lattice QCD results in thermodynamic equilibrium (DQPM). The DQPM describes QCD properties in terms of single-particle Green's functions (in the sense of a two-particle irreducible approach) and leads to the notion of the constituents of the sQGP being effective quasiparticles, which are massive and have broad spectral functions (due to large interaction rates). The transition from partonic to hadronic degrees of freedom in PHSD is described by covariant transition rates for the fusion of quark-antiquark pairs to mesonic resonanses or three quarks (antiquarks) to baryonic states.

Dilepton radiation by the constituents of the QGP proceeds via the elementary processes illustrated in Fig. [: the Born (Drell-Yan) $q+\bar{q}$ annihilation mechanism, Gluon Compton scattering $\left(q+g \rightarrow \gamma^{*}+q\right.$ and $\left.\bar{q}+g \rightarrow \gamma^{*}+\bar{q}\right)$, and quark + anti-quark annihilation with gluon Bremsstrahlung in the final state $\left(q+\bar{q} \rightarrow g+\gamma^{*}\right)$. In the on-shell approximation, one uses perturbative QCD cross sections for the processes listed above. However, in order to make quantitative predictions at experimentally relevant low dilepton mass and strong coupling, we have to take into account the nonperturbative spectral functions and self-energies of the quarks, anti-quarks and gluons thus going beyond the on-shell approximation. For this purpose, off-shell cross sections were derived in [ए6] for dilepton production in the reactions $q+\bar{q} \rightarrow l^{+} l^{-}$(Drell-Yan mechanism), $q+\bar{q} \rightarrow g+l^{+} l^{-}$ (quark annihilation with the gluon Bremsstrahlung in the final state), $q(\bar{q})+g \rightarrow q(\bar{q})+l^{+} l^{-}$(gluon Compton scattering), $g \rightarrow q+\bar{q}+l^{+} l^{-}$and $q(\bar{q}) \rightarrow q(\bar{q})+g+l^{+} l^{-}$(virtual gluon decay, virtual 


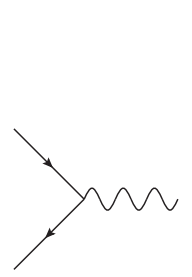

(a)

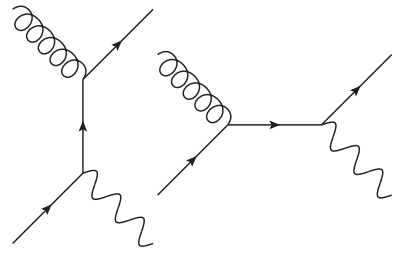

(b)

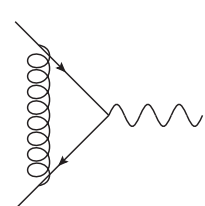

(c)

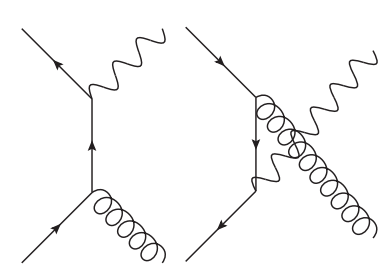

$(d)$

Figure 1: Diagrams contributing to the dilepton production from a QGP: (a) Drell-Yan mechanism, (b) gluon-Compton scattering (GCS), (c) vertex correction, (d) gluon Bremsstrahlung (NLODY), where virtual photons (wavy lines) split into lepton pairs, spiral lines denote gluons, arrows denote quarks. In each diagram the time runs from left to right.

quark decay) in the sQGP in effective perturbation theory by dressing the quark and gluon lines with the DQPM propagators for quarks and gluons. The obtained off-shell elementary cross sections then are implemented into the PHSD transport code, where the masses of quarks and gluons are distributed according to the DQPM spectral functions.

For illustration, we plot the dilepton production cross sections in the Born mechanism on the 1.h.s. of Fig. \. The short dashes (black) line shows the on-shell, i.e. the standard perturbative result. The other lines show the off-shell cross section, in which the annihilating quark and antiquark have finite masses $m_{1}$ and $m_{2}$ with different values: $m_{1}=0.3 \mathrm{GeV}, m_{2}=0.15 \mathrm{GeV}$ (solid magenta line), $m_{1}=0.3 \mathrm{GeV}, m_{2}=0.3 \mathrm{GeV}$ (dash-dotted red line), $m_{1}=0.3 \mathrm{GeV}, m_{2}=0.6 \mathrm{GeV}$ (dashed blue line), $m_{1}=0.6 \mathrm{GeV}, m_{2}=0.6 \mathrm{GeV}$ (dash-dot-dot green line).

In Fig. [1], the off-shell cross sections for the quark annihilation with gluon bremsstrahlung channel at various values of quark and gluon off-shellnesses (masses) are compared to the on-shell (pQCD) result. Dashed black line shows the on-shell cross section regularized by a cut on gluon mass $\mu_{\text {cut }}=0.206 \mathrm{GeV}$, red solid line presents the off-shell cross section for the gluon mass fixed to $\mu=0.8 \mathrm{GeV}$ and on-shell quark and anti-quark $\left(m_{1}=m_{2}=m_{3}=0\right)$. Blue dash-dotted line gives the off-shell result for $\mu=0.8 \mathrm{GeV}, m_{1}=m_{2}=m_{3}=m_{q}=0.6 \mathrm{GeV}$. One readily notices the shift of the maximum allowed mass of the lepton pair to a lower value (in order to produce a massive gluon in the final state).

\section{Comparison to data at SPS energies}

By employing the HSD approach to the low mass dilepton production in relativistic heavy-ion collisions, it was shown in [ए7] that the NA60 Collaboration data for the invariant mass spectra for $\mu^{+} \mu^{-}$pairs from In+In collisions at $158 \mathrm{~A} \cdot \mathrm{GeV}$ favored the 'melting $\rho$ ' scenario [एप]]. Also the data from the CERES Collaboration [20] showed a preference for the 'melting $\rho$ ' picture. On the other hand, the dilepton spectrum from In+In collisions at $158 \mathrm{~A} \cdot \mathrm{GeV}$ for $M>1 \mathrm{GeV}$ could not be accounted for by the known hadronic sources (see Fig.2 of [ㅁ]]). Also, hadronic models do not reproduce the softening of the $m_{T}$ distribution of dileptons at $M>1 \mathrm{GeV}$ [ए耳]. This observation pointed towards a partonic origin of the spectra at $M>1 \mathrm{GeV}$.

In Ref. [2]] we presented PHSD results for the dilepton spectrum as produced in $I n+I n$ reactions at $158 \mathrm{AGeV}$ compared to the NA60 data [[प, [2]]. We found that the spectrum at invariant 


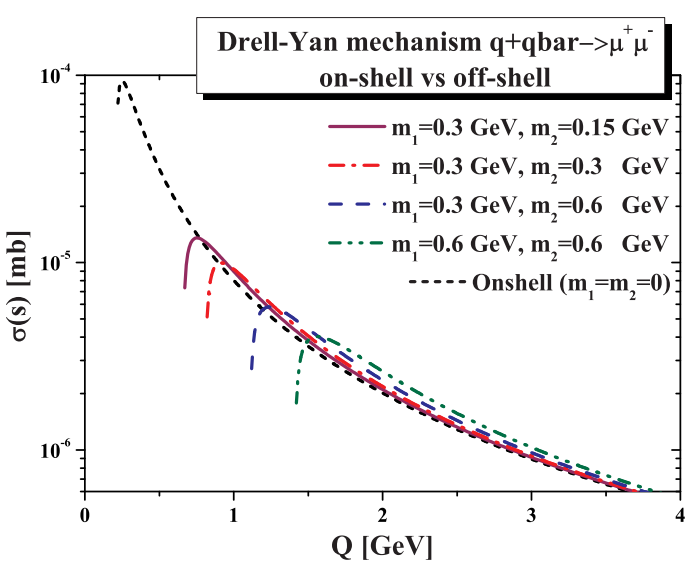

Figure 2: Dilepton production cross sections in the Born channel $\left(q+\bar{q} \rightarrow \mu^{+}+\mu^{-}\right)$. The short dashes (black) line shows the on-shell, i.e. the standard pQCD result. The other lines show the off-shell cross section, in which the annihilating quark and antiquark have finite masses $m_{1}$ and $m_{2}$ with different values (see legend).

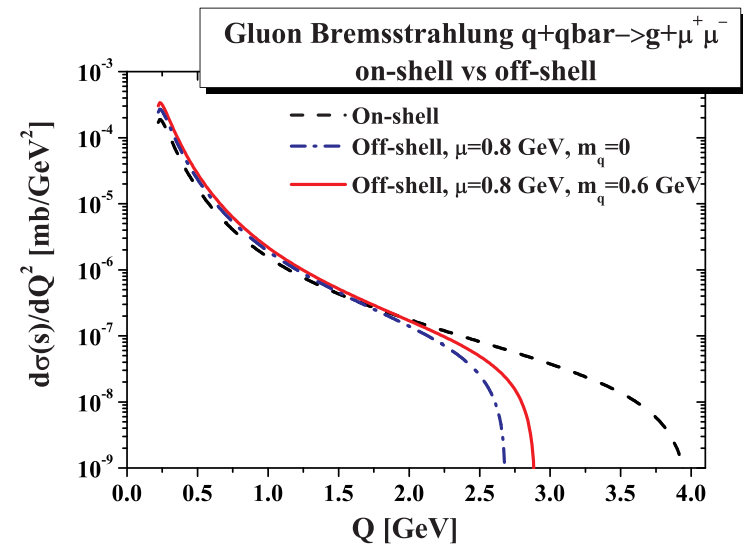

Figure 3: Comparison of off-shell and on-shell cross sections for dilepton production in the gluon Bremsstrahlung $q+\bar{q} \rightarrow g+\mu^{+} \mu^{-}$channel at $\sqrt{s}=$ $4 \mathrm{GeV}$. Dashed black line shows the on-shell (pQCD) cross section regularized by the cut on gluon mass $\mu_{\text {cut }}=0.206 \mathrm{GeV}$, blue dash-dotted and red solit lines present the off-shell cross section for the finite parton masses.

masses below $1 \mathrm{GeV}$ was well reproduced by the $\rho$ meson yield, if a broadening of the meson spectral function in the medium was assumed. On the other hand, the spectrum at $M>1 \mathrm{GeV}$ was shown to be dominated by the partonic sources. Moreover, accounting for partonic dilepton sources allowed to reproduce in PHSD the effective temperature of the dileptons (slope parameters) in the intermediate mass range. The softening of the transverse mass spectrum with growing invariant mass implies that the partonic channels occur dominantly before the collective radial flow has developed.

\section{Comparison to data at RHIC energies}

The PHENIX Collaboration has presented dilepton data from $p p$ and $A u+A u$ collisions at Relativistic-Heavy-Ion-Collider (RHIC) energies of $\sqrt{s}=200 \mathrm{GeV}$ [23], 24] which show a large enhancement in $A u+A u$ reactions (relative to scaled $p p$ collisions) in the invariant mass regimes from 0.15 to $0.6 \mathrm{GeV}$ and from 1 to $4 \mathrm{GeV}$ [[2]]. We recall that HSD provides a reasonable description of hadron production in $A u+A u$ collisions at $\sqrt{s}=200 \mathrm{GeV}$ [[26] and present in Figs 目 and [5] the HSD results for $e^{+} e^{-}$pairs in inclusive $A u+A u$ collisions in comparison to the data from PHENIX [24] as calculated in [ㅁ]]. Whereas the total yield is quite well described in the region of the pion Dalitz decay as well as around the $\omega$ and $\phi$ mass, HSD clearly underestimates the measured spectra in the regime from 0.2 to $0.6 \mathrm{GeV}$ by an average factor of 3 . When including the in-medium modification scenarios for the vector mesons, we achieve a sum spectrum which is only slightly enhanced compared to the 'free' scenario. The low mass dilepton spectra from $A u+A u$ collisions at RHIC (from the PHENIX Collaboration) are clearly underestimated in the invariant mass range from 0.2 to $0.6 \mathrm{GeV}$ in the 'collisional broadening' scenario as well as in the 'dropping mass + collisional broadening' model. We mention that HSD results for the low mass dileptons 


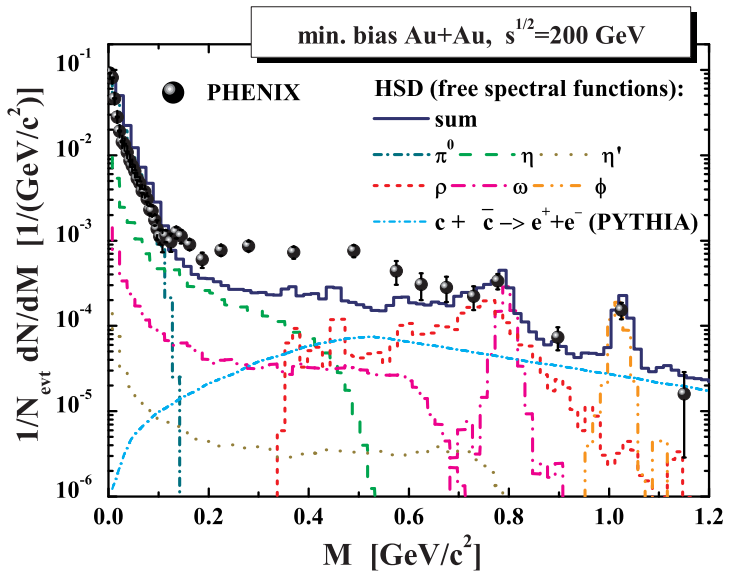

Figure 4: The HSD results for the mass differential dilepton spectra in case of inclusive $A u+A u$ collisions at $\sqrt{s}=200 \mathrm{GeV}$ in comparison to the data from PHENIX [24]. The results are shown for vacuum spectral functions (for $\rho, \omega, \phi$ ) including the channel decompositions.

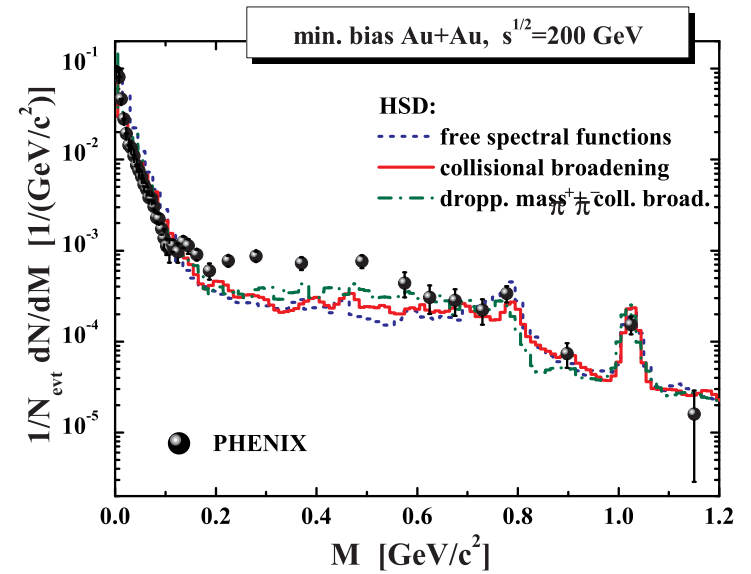

Figure 5: The HSD results for the mass differential dilepton spectra in case of inclusive $A u+A u$ collisions at $\sqrt{s}=200 \mathrm{GeV}$ in comparison to the data from PHENIX [R4] in the 'free' scenario, the 'collisional broadening' picture as well as the 'dropping mass + collisional broadening' model.

are very close to the calculated spectra from van Hees and Rapp as well as Dusling and Zahed [27] (cf. the comparison in Ref. [R8]). At higher masses (from 1 to $4 \mathrm{GeV}$ ) the only hadronic sources of correlated lepton pairs are the charmed mesons: semi-leptonic decays of correlated D-mesons and the dilepton decays of charmonia. Between the $\phi$ and $J / \Psi$ peaks, the HSD results underestimate the PHENIX data by approximately a factor of two. In analogy to the analysis of the data at SPS energies in the previous Section, we attribute the missing yield to partonic contributions.

By implementing the off-shell partonic processes into the PHSD transport approach, we calculate the dilepton spectra in $A u+A u$ at $\sqrt{s}=200 \mathrm{GeV}$ and compare to the PHENIX data in Figs. 6 and प. In Fig. G we present the low mass region $(M=0-1.2 \mathrm{GeV})$; in this region, the yield in PHSD is dominated by hadronic sources and essentially coincides with the HSD result. There is a discrepancy between the PHSD calculations and the data in the region of masses from 0.2 to $0.6 \mathrm{GeV}$. The discrepancy is not amended by accounting for the radiation from the QGP, since the latter is 'overshone' by the radiation from hadrons integrated over the evolution of the collision. In contrary, the partonic radiation is visible in the mass region $M=1-4 \mathrm{GeV}$, as on sees in Fig. $\square$. Dileptons generated by the quark-antiquark annihilation in sQGP constitute about a half of the observed yield in the mass range between the masses of the $\phi$ and the $J / \Psi$ mesons. At $M>2.5 \mathrm{GeV}$, the partonic yield dominates over the D-meson contribution. Thus, accounting for partonic radiation in PHSD fills up the gap between the hadronic model results [177, 25] and the data at $M>1 \mathrm{GeV}$.

\section{Summary}

Parton Hadron String Dynamics [Q] (PHSD) transport approach incorporates the relevant offshell dynamics of the vector mesons as well as the explicit partonic phase in the early hot and dense reaction region. Comparison of the transport calculations to the data of the CERES and NA60 Collaborations points towards 'melting' modification of the $\rho$ '-meson at high densities, i.e. the 


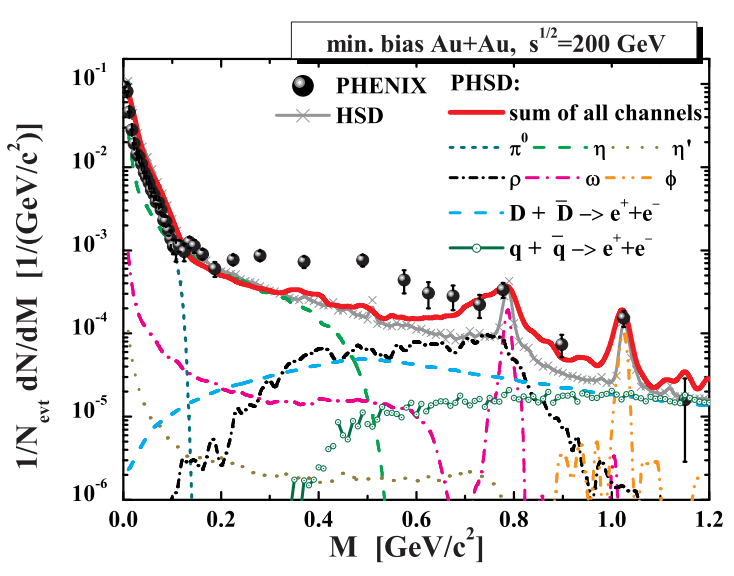

Figure 6: The PHSD results for the mass differential dilepton spectra in case of inclusive $A u+A u$ collisions at $\sqrt{s}=200 \mathrm{GeV}$ in comparison to the data from PHENIX [24] in the low mass region $(M=$ $0-1.2 \mathrm{GeV})$. For comparison, the HSD results are shown by the grey line with cross symbols.

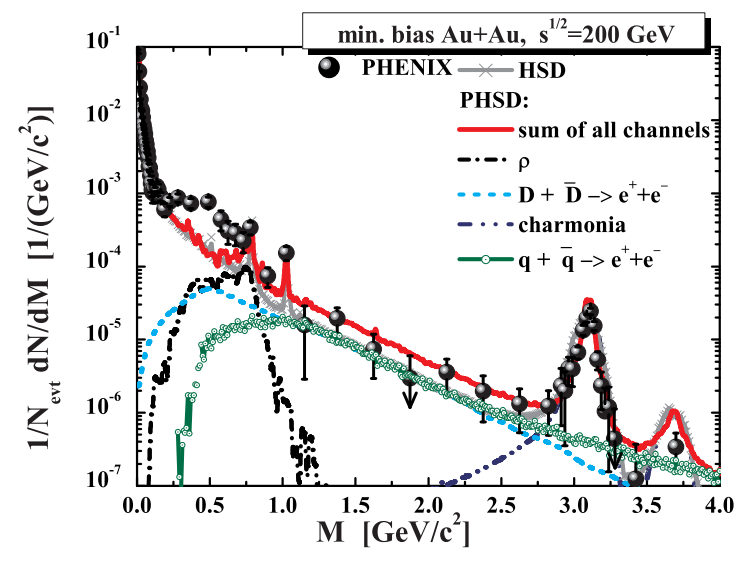

Figure 7: The HSD results for the mass differential dilepton spectra in case of inclusive $A u+A u$ collisions at $\sqrt{s}=200 \mathrm{GeV}$ in comparison to the data from PHENIX [24] for $M=0-4 \mathrm{GeV}$. For comparison, the HSD results are shown by the grey line with cross symbols.

broadening of the vector meson's spectral function. On the other hand, the spectrum at $M>1 \mathrm{GeV}$ is shown to be dominated by the partonic sources.

The low mass dilepton spectra from $A u+A u$ collisions at RHIC (from the PHENIX Collaboration) are clearly underestimated by the hadronic channels in the invariant mass range from 0.2 to $0.6 \mathrm{GeV}$. The discrepancy is not amended by accounting for the radiation from the QGP, since the latter is 'over-shone' by the radiation from hadrons integrated over the evolution of the collision.

In contrary, the partonic radiation is visible in the mass region $M=1-4 \mathrm{GeV}$. Dileptons generated by the quark-antiquark annihilation in sQGP constitute about a half of the observed yield in the mass range between the masses of the $\phi$ and the $J / \Psi$ mesons. At $M>2.5 \mathrm{GeV}$, the partonic yield dominates over the D-meson contribution. Thus, accounting for partonic radiation in PHSD fills up the gap between the hadronic model results [177, [25] and the data at $M>1 \mathrm{GeV}$.

A detailed calculation of the dilepton production in heavy ion collisions at RHIC energy versus collision centrality, dilepton mass $M$ and transverse momentum $p_{T}$ will be published in near future. The comparison to the dilepton data will open the possibility to study the relative importance of different processes in the dilepton production and guide us towards a better understanding of the properties of matter created in heavy-ion collisions.

\section{Acknowledgments}

Work supported in part by the "HIC for FAIRŤ framework of the "LOEWEŤ program. We acknowledge stimulating discussions with S. Damjanovic, J.Manninen, A. Toia. 


\section{References}

\section{References}

[1] E. V. Shuryak, Sov. Phys. JETP 47, 212 (1978).

[2] E. V. Shuryak, Phys. Lett. B78, 150 (1978), Sov.J.Nucl.Phys. 28 (1978) 408, Yad.Fiz. 28 (1978) 796.

[3] E. L. Feinberg, Izv. Akad. Nauk Ser. Fiz. 34, 1987 (1970).

[4] E. L. Feinberg, Nuovo Cim. A34, 391 (1976).

[5] J. D. Bjorken and H. Weisberg, Phys. Rev. D13, 1405 (1976).

[6] G. Agakichiev et al., CERES Collaboration, Phys. Rev. Lett. 75 (1995) 1272; Th. Ullrich et al., Nucl. Phys. A610 (1996) 317c; A. Drees, Nucl. Phys. A610 (1996) 536c.

[7] M. A. Mazzoni, HELIOS Collaboration, Nucl. Phys. A566 (1994) 95c; M. Masera, Nucl. Phys. A590 (1995) 93c; T. Åkesson et al., Z. Phys. C68 (1995) 47.

[8] O. Linnyk, E. L. Bratkovskaya and W. Cassing, Nucl. Phys. A 830 (2009) 491C.

[9] W. Cassing and E. L. Bratkovskaya, Phys. Rev. C 78 (2008) 034919, Nucl. Phys. A 831 (2009) 215.

[10] W. Cassing, E. L. Bratkovskaya, Phys. Rept. 308 (1999) 65.

[11] E. L. Bratkovskaya, W. Cassing, Nucl. Phys. A619 (1997) 413.

[12] W. Ehehalt, W. Cassing, Nucl. Phys. A 602 (1996) 449.

[13] W. Cassing, S. Juchem, Nucl. Phys. A 665 (2000) 377; ibid. A 672 (2000) 417.

[14] E. L. Bratkovskaya, W. Cassing, Nucl. Phys. A 807 (2008) 214.

[15] J. Ruppert, T. Renk and B. Müller, Phys. Rev. C 73 (2006) 034907.

[16] O. Linnyk, arXiv:1004.2591

[17] E. L. Bratkovskaya, W. Cassing and O. Linnyk, Phys. Lett. B 670 (2009) 428.

[18] W. Cassing, Nucl. Phys. A 791 (2007) 365; ibid. A 795 (2007) 70.

[19] R. Arnaldi et al., NA60 Collaboration, Phys. Rev. Lett. 96 (2006) 162302; J. Seixas et al., J. Phys. G 34 (2007) S1023; S. Damjanovic et al., Nucl. Phys. A 783 (2007) 327c.

[20] D. Adamova et al. CERES Collaboration, Nucl. Phys. A 715 (2003) 262; Phys. Rev. Lett. 91 (2003) 042301; G. Agakichiev et al., Eur. Phys. J. C 41 (2005) 475; D. Adamova et al. Phys. Lett. B 666 (2008) 425; A. Marin et al.; Proceedings of CPOD07, PoS 034 (2007).

[21] O. Linnyk, E. L. Bratkovskaya and W. Cassing, Nucl. Phys. A 830 (2009) 491C, O. Linnyk, E. L. Bratkovskaya and W. Cassing, arXiv:1001.3858 [nucl-th].

[22] R. Arnaldi et al., NA60 Collaboration, Eur. Phys. J. C 59, 607 (2009)

[23] A. Adare et al., PHENIX Collaboration, Phys. Lett. B 670 (2009) 313

[24] A. Toia et al., PHENIX Collaboration, Nucl. Phys. A 774 (2006) 743; Eur. Phys. J 49 (2007) 243; S. Afanasiev et al., PHENIX Collaboration, arXiv:0706.3034 [nucl-ex]; A. Adare et al., PHENIX Collaboration, Phys. Rev. C 81 (2010) 034911.

[25] J. Manninen, E. L. Bratkovskaya, W. Cassing and O. Linnyk, arXiv:1005.0500 [nucl-th]. 
[26] E. L. Bratkovskaya, W. Cassing, H. Stöcker, Phys. Rev. C 67 (2003) 054905; E. L. Bratkovskaya et al. Phys. Rev. C 69 (2004) 054907.

[27] K. Dusling and I. Zahed, Nucl. Phys. A 825 (2009) 212.

[28] A. Toia, J. Phys. G 35 (2008) 104037. 